\title{
Frontières
}

\section{La mort du colonel}

\section{Georges Anglade}

Volume 16, numéro 1, automne 2003

Remède ou poison?

URI : https://id.erudit.org/iderudit/1073769ar

DOI : https://doi.org/10.7202/1073769ar

Aller au sommaire du numéro

Éditeur(s)

Université du Québec à Montréal

ISSN

1180-3479 (imprimé)

1916-0976 (numérique)

Découvrir la revue

Citer ce document

Anglade, G. (2003). La mort du colonel. Frontières, 16(1), 95-97.

https://doi.org/10.7202/1073769ar

Ce document est protégé par la loi sur le droit d'auteur. L'utilisation des services d'Érudit (y compris la reproduction) est assujettie à sa politique d'utilisation que vous pouvez consulter en ligne.

https://apropos.erudit.org/fr/usagers/politique-dutilisation/
Cet article est diffusé et préservé par Érudit.

Érudit est un consortium interuniversitaire sans but lucratif composé de l’Université de Montréal, l'Université Laval et l'Université du Québec à Montréal. Il a pour mission la promotion et la valorisation de la recherche. https://www.erudit.org/fr/ 


\section{LA MORT DU COLONEL}

Georges Anglade,
écrivain.

L'une de mes lodyans, que j'ai intitulée «La mort du colonel», traite de l'usage du poison en Haiti. La voici :

Nous étions plusieurs à rouler normalement, en cette fin d'un dimanche, les derniers kilomètres de la côte aux plages, à l'est de Port-au-Prince, quand toutes les radios interrompirent leur programme pour lire le communiqué : le Colonel le plus puissant de l'heure s'était fait avoir, chez lui, dans sa soupe jaune du matin. Empoisonné. Mort. Raide. Et ce fut la plus folle course jamais vue dans Port-au-Prince. À pied ou en voiture, tout le monde fonçait vers sa demeure ou un abri quelconque. L'on devait rapporter plus tard des choses incroyables: des voitures se rentraient dedans et les conducteurs affables renvoyaient, sans s'arrêter, les formalités au lendemain. Si Dieu le veut! Trois paralytiques reconnaissants répétèrent à l'envi toute la semaine à tous les micros qu'ils s'étaient levés d'un coup pour courir sans bâton ni béquilles. Miracle! Moins d'une heure après le communiqué, et il était à peine cinq heures de l'aprèsmidi, les rues habituellement noires de monde étaient blanches. Pas le moindre signe de vie. Une station de radio évoqua les conséquences publiques de l'assassinat de l'Archiduc à Sarajevo... et une autre les conséquences privées de la grande panne d'électricité de New York... mais aucune n'insista sur la soupe jaune. Les centrales téléphoniques surchargées étaient tombées en panne et la brusque demande d'électricité avait fait sauter les systèmes. Port-auPrince était dans le noir et le silence. La veillée forcée qui commençait allait être longue. Une ville entière s'était terrée et retenait son souffle dans l'attente, jusqu'au petit matin, de suites apocalyptiques. Mais il ne se passa rien. Absolument rien. En apparence du moins. Car.
Il fallait remonter au temps de l'esclavage et de l'indépendance, où les poisons furent des armes quotidiennes, pour saisir que toutes ces pratiques n'avaient certainement pas disparu d'un coup dans la période nationale. Aussi, la première de toutes les sages craintes entretenues d'une génération à l'autre, était-elle justement celle de l'empoisonnement. Il fallait, hors de chez soi, s'en garder continuellement par des manières adéquates. Mais il fallait aussi mettre un point d'honneur à ce que sa maison en soit exempte, poison free, disait-on même des maisons honnêtes, pour les distinguer. Et comme la notion de mort naturelle n'a jamais eu cours à Quina, c'est évidemment dès l'enfance que je fus exposé, comme tout le monde, aux raffinements des histoires qui dressaient un catalogue exhaustif des substances et de leur mode d'emploi, dont deux ou trois avaient particulièrement frappé mes jeunes âges.

Il s'était même greffé, sur l'ensemble des convenances courantes, des façons particulières de recevoir quand on faisait de la politique. Or ils en faisaient tous, du matin au soir, à commenter les nouvelles du jour, à propager les rumeurs les plus folles, à en créer même à l'occasion quand les événements nouveaux se faisaient rares. Au point que tout devint politique et que pour n'importe quel étranger en visite, on mettait en branle ce code. On ne pouvait ainsi offrir à boire du rhum à un ayant droit, élu ou nommé, fonctionnaire ou militaire, héritier ou homo novus - beaucoup de monde, beaucoup de monde - qu'à partir d'une bouteille neuve hermétiquement bouchée avec son cachet d'origine. Elle devait être ostensiblement ouverte avec quelque difficulté, feinte ou réelle, sous les yeux de l'invité qui devait à son tour feindre ne pas s'en apercevoir, ou mieux, protester après coup qu'une bouteille déjà ouverte eut très bien fait l'affaire. J'ai souvent entendu dire: Nous n'avons plus de rhum, quand il y avait sur les étagères deux à trois bouteilles à peine entamées. C'était pour dire: Nous n'avons plus de bouteille neuve à déboucher pour un hôte de passage.

Ainsi Quina s'enfonçait-elle lentement dans une psychose du poison avec d'étranges politesses et bonnes manières d'exorcismes. Tous les gestes étaient codifiés comme autant d'antidotes à la panoplie des poisons. Il fallait par exemple toujours raccompagner un visiteur jusqu'aux lisières de sa propriété si la brune du soir était déjà tombée. C'est que l'une des plus redoutables substances ne faisait effet que si celui qui l'avait absorbée s'exposait ensuite au serein. C'était le fameux Pinga serein, le Prends garde au serein! On pouvait donc convivialement boire la potion avec son invité et le laisser partir seul, disparaître dans le noir. Et que dire du café, que tout grand amateur de café ne boit jamais que chez lui, sous prétexte de savants dosages personnels des poudres, mais plus prosaïquement parce qu'il est bien connu que le fond d'une tasse de café est noir assez pour cacher n'importe quelle substance. Anba kafe fè nwa!

Même si l'on bassinait les jeunes avec des préceptes de base d'une bonne éducation, "À votre service » c'est l'usage, "Non merci " c'est façon -, la langue de Quina avait gardé quelques tournures anciennes comme pour dire que la politesse et les bonnes manières sont choses qui traversent le temps sans rides -, il s'était aussi développé une brochette de rituels d'amicale confiance et de sincères alliances dont la plus significative était le partage de la soupe jaune. En toutes circonstances notables, comme le $1^{\text {er }}$ janvier ou le jour anniversaire du père de famille, ou au plus vénéré moment de la semaine, le dimanche matin, ou pour les grands départs et les grandes retrouvailles... la soupe jaune au menu était hautement symbolique. Chaque maîtresse de maison avait sa recette particulière et l'on savait à l'avance le registre des assaisonnements que l'on trouverait à sa table 
et le fumet qui distinguerait sa soupe jaune de toutes les autres, comme une signature. C'était même la seule invitation qui dispensait de toutes les formalités de protection, on y buvait goulûment tous les restes de bouteilles ouvertes, sirotait café sur café, et les convives gagnaient allègrement seuls la nuit sans être raccompagnés.

Bien que tous les petits provinciaux eussent été régulièrement sermonnés sur les perfidies port-au-princiennes et mis en garde contre toutes les trahisons qui s'y déroulaient, jamais au grand jamais n'avait-on évoqué la soupe jaune comme pouvant faire un jour partie des profanations de la capitale.

Cette lodyans n'est que l'une des illustrations littéraires de la présence significative des références au poison dans beaucoup des histoires racontées tout au long du $\mathrm{XX}^{\mathrm{e}}$ siècle par trois générations d'écrivains haïtiens. Notamment les lodyanseurs. J'y ai moi-même fait allusion dans cinq à six de mes histoires, avant d'aborder le thème de front dans La mort du colonel. C'est que le poison a fait partie des matières au programme de l'enfance de ma génération de provincial, élevé à Port-au-Prince dans les années 1950 ; et j'ai su très tôt que cela datait de deux siècles exactement, 1750, puisqu'on disait encore indifféremment $\mathrm{du}$ makandal pour du poison et que l'on désignait encore par un makandal ou une makandale l'empoisonneur ou l'empoisonneuse au tribunal de paix d'Aquin et que la cérémonie vodou de préparation de poisons, de sortilèges, de viatiques, et autres « paquets » est dite un makandal dans mon coin de pays. En ce temps, il fallait une autorisation en bonne et due forme de la préfecture d'Aquin sur une fiche cartonnée rose orangée pour les cérémonies publiques du vodou comme un "manger blanc», mais personne n'était jamais venu parler de cérémonies secrètes et privées du makandal. Et pourtant. Les grandes personnes en parlaient en dodelinant un oui de la tête de manière convenue.

L'affaire Makandal est la première menace globale de la colonie de Saint-Domingue et c'est par le poison que son instigateur comptait éliminer tous les Blancs de l'île. Cette accusation contre lui est portée dès le début de l'affaire. L'action projetée, identique dans son intention avec celle qui réussira cinquante ans plus tard par l'indépendance, est donc une vaste opération d'empoisonnement comme jamais plus après on n'en verra à cette échelle dans la colonie et même ailleurs aux Amériques. Le premier héros national haïtien est donc ce Makandal et la première arme nationale haiitienne a été le poison. Plus tard joueront

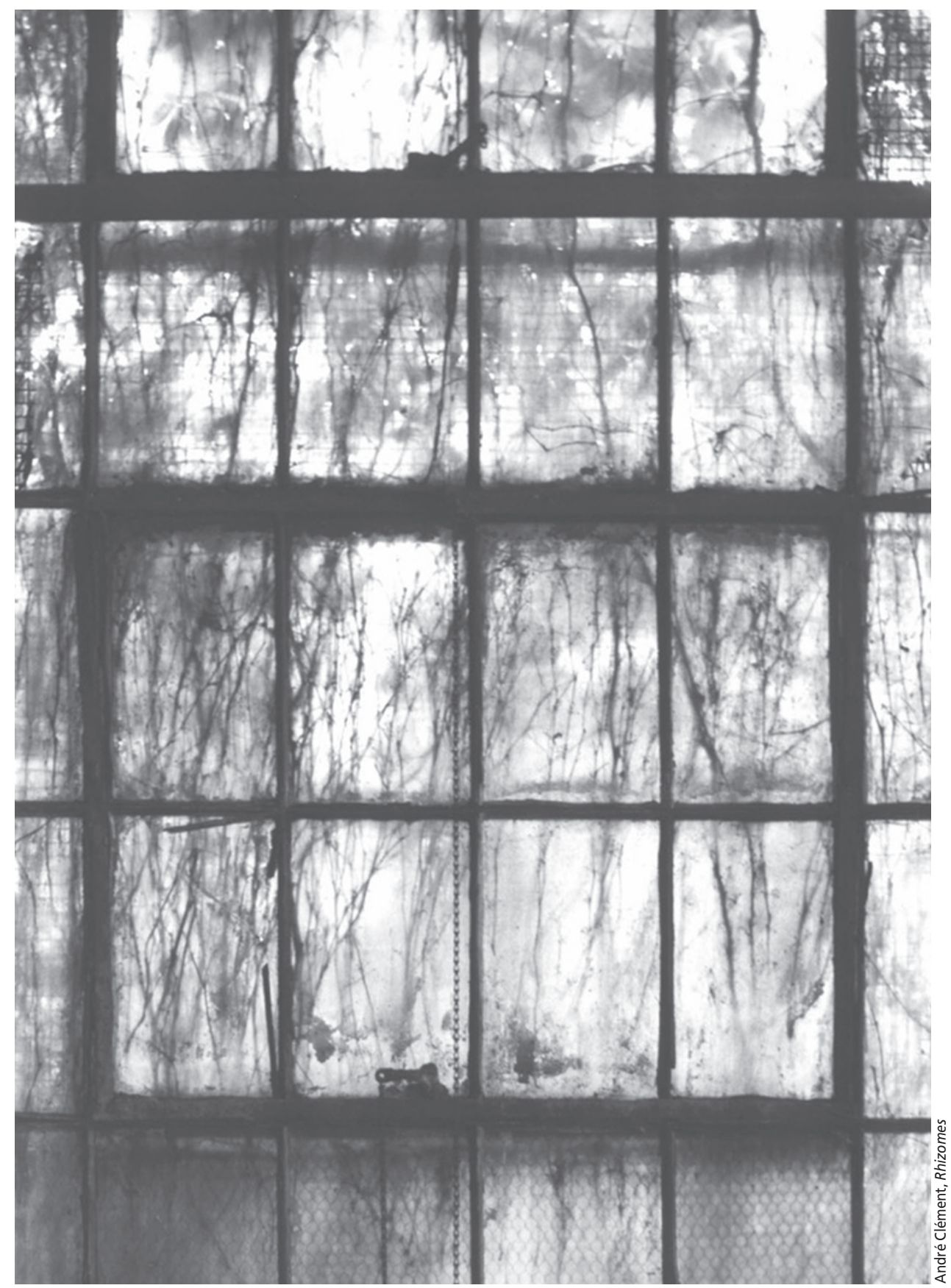

le vodou et Boukman, le marronnage et les bandes rivales, les grenadiers à l'assaut, les généraux noirs et mulâtres, le créole et la lodyans, et caetera. Mais à l'origine, avant que tout le reste ne prenne corps en culture de résistance nationale par la langue (créole), le système de croyance (vodou), la manière narrative (lodyans), les façons culturales (compagnonnage)... il y eut cette première arme de combat: le poison. Et cela se comprend bien, car le niveau d'organisation encore embryonnaire obligeait à un recours de ce type pour arriver à menacer l'existence même de la colonie; du moins telle fut la crainte des colons. Et voilà le poison promu première arme de terreur et de destruction massive proprement haïtienne dans son projet de libération!
Sur François Makandal, la littérature et la peinture ont maintenant deux siècles et demi d'effervescences. Moreau de SaintMéry raconte dans sa Description avoir acheté à Versailles, avant 1789 forcément, le portrait de Makandal en prison par le peintre parisien Dupont. Comme Makandal ne devait passer que les nuits des 18 et 19 janvier 1758 en prison, l'on devrait pouvoir dater très précisément ce tableau et s'approcher au maximum de ce à quoi il devait ressembler. C'était un marabout, comme on en trouve encore à Fond-desBlancs de la descendance des brigades polonaises qui prirent cause haïtienne à l'indépendance: traits fins au nez aquilin, peau d'un noir soutenu, cheveux droits de jais, face émaciée. Des Éthiopiens pour faire 
image et pour reprendre la parole courante dans cette zone que le mélange polonais et noir donne du marabout éthiopien. On dit, mais on en a tellement dit sur Makandal, qu'il est seulement certain que c'était un bossale, c'est-à-dire né en Afrique; mais qu'il ait été un musulman lettré parlant et écrivant l'arabe participe de sa mythologie spontanée et immédiate dès la deuxième moitié du XVIII ${ }^{\mathrm{e}}$ siècle à un tel point que le Mercure de France du 15 septembre 1787 lui consacre un conte! La consécration du premier héros à la statue tenant une fiole pour arme!

Il est surtout certain que Makandal connaissait les poisons à faire frémir la colonie et à mobiliser les nègres dans son projet. Il avait vécu au Limbé sur la côte nord sucrière et devait être un nègre-àtalent pour qu'un jour à alimenter le moulin à canne, sa main se prenne dans les rouages et qu'il fallut la lui couper avant que le reste du corps ne suive dans les engrenages. Le Normand de Mézy, son propriétaire, l'affectera au gardiennage des bêtes à cornes et cavales d'où il marronnera pour semer une juste terreur jusqu'au bûcher du 20 janvier 1758. Cette toute première figure d'envergure nationale, régionale, continentale même, comme le seront après Toussaint, Jose Marti, Guevara... va empoisonner la relation entre maîtres et esclaves, grande case et cases-à-nègres, dans toute l'Amérique esclavagiste à partir de cette affaire de poisons.

Alejo Carpentier dans Le Royaume de ce monde, et Édouard Glissant dans Monsieur Toussaint feront une place centrale au personnage de Makandal qui sera même, dans ce dernier ouvrage, le juge des actes de nul autre que Toussaint pour son éventuelle intronisation au panthéon des mythes. Et Makandal, c'est le poison sous toutes ses formes telles que l'on va en hériter dans la période nationale avec l'admiration due à son rang d'arme de guerre d'indépendance mais aussi la terreur qu'il inspire à le voir s'insinuer au quotidien des relations.

La panoplie des poisons est certainement vaste et reliée aux savoir-faire propres à chaque région car il faut toujours se représenter ce pays, jusqu'aux années 1950 du triomphe définitif de la centralisation, comme une mosaïque séculaire de onze républiques distinctes aux onze ports d'exportations et aux onze façons régionales particulières dans à peu près toutes les manifestations de la même culture de base, paysanne avec intermédiaires exportateurs de denrées sis dans les bourgs. Aussi de la culture du poison je ne connais en fait, et seulement un peu, que celle de ma province quinoise, mais il me paraît que le phénomène de la zombification traverse à peu près tout le pays, même s'il n'a dû fleurir qu'en des zones bien précises. C'est l'arme du poison portée à son plus haut niveau dans des recettes secrètes capables de provoquer la catatonie mortuaire suivie d'un réveil après inhumation. René Dépestre en a fait son fond dans Hadriana dans tous mes rêves mais aussi Frankétienne dans Dézafi pour dire que tous les écrivains indistinctement ont touché au phénomène. Punition sévère sociale ? Captation économique de main-d'œuvre ? Épisodes extrêmes de processus conflictuels ? Rituels ?... Une équation de tout cela certainement avec des pondérations variables de chaque facteur explicatif en fonction de la période, de la région, etc. Ce sera cela la contribution des scientifiques à s'être penchés sur le phénomène, comme Emerson Douyon de l'Université de Montréal s'était penché voilà une vingtaine d'années sur un cas très médiatisé dans l'Artibonite pour son étrangeté. Une équipe de l'université Harvard avait aussi traqué la formule secrète haïtienne avec acharnement jusqu'à la trouver semble-t-il... Et ne voilà-t-il pas qu'en ce mois de septembre 2003, la très sérieuse NASA américaine fait état d'expériences de «retour de la mort» pour ses besoins d'exploration par navettes habitées en se référant calmement et le plus sérieusement du monde à la formule de la zombification haïtienne. Tout Haïti chuchotait, j'en arrive justement.

On connaît finalement très peu de la culture tricentenaire haïtienne mais assez pour problématiser le poison autrement qu'en une simple ingestion de produits toxiques à des doses létales. La zombification est là pour en témoigner et son imprégnation dans la culture est telle que l'on empoisonne encore aujourd'hui la dépouille des décédés pour empêcher toute réutilisation éventuelle de la personne à d'autres fins après usage de l'antidote. Une honorable pompe funèbre de Port-au-Prince vient de subir, ce mois-ci de septembre 2003, les assauts d'une foule déchaînée réclamant un cadavre qui aurait semblet-il appelé la veille au soir les siens par téléphone cellulaire pour se dire bien en vie dans la morgue. Le bilan des dommages est sévère: entre autres, deux corbillards détruits à la une des nouvelles locales.

Et puis le plus violent de tous les poisons, et le plus utilisé dans la littérature de la vengeance quotidienne, est le «Trois dégouttes » dont le côté lugubre de la cueillette a de quoi révulser même le lecteur amateur de frissons. Il suffit de retourner un cadavre en processus de décomposition depuis au moins une journée et de recueillir trois gouttes du fluide corporel à s'échapper de ses lèvres pour avoir une potion magique dont personne ne peut échapper : aucun antidote connu à ce jour et la mithridatisation implique un tel dégoût qu'il vaut mieux encore en mourir. La littérature fantastique y a trouvé une arme absolue.

De quoi est mort le colonel? Avant même du contenu de la fiole, c'est d'une violente profanation de la convivialité de la soupe jaune. C'est que de toutes les armes mortelles, le poison ne serait-il pas le plus terrifiant pour l'autre par l'obligation de le lui faire absorber au moment hautement symbolique du boire et du manger? Makandal a inventé la première arme de destruction massive, sinon du colon luimême du moins de son imaginaire par la terreur de gestes quotidiens.

\section{Bibliographie}

CARPENTIER, Alejo (1948). Le Royaume de ce monde. Paris, Folio.

DÉPESTRE, René (1988). Hadriana dans tous mes rêves. Paris, Gallimard.

FRANKÉTIENNE [FRANCK Etienne] (1975). Dézafi, Port-au-Prince, Éditions Fardin.

GLISSANT, Édouard (1958). Monsieur Toussaint. Paris, Gallimard.

MOREAU DE SAINT MÉRY, Médéric-Louis-Élie (1958). Description topographique, physique, civile, politique et historique de la partie française de l'île de Saint-Domingue. 1796. Paris.

\section{Note}

1. Lodyans extraites de Leurs jupons dépassent, PLC, Petite Collection Lanctôt, $4^{\mathrm{e}}$ trimestre 2003 (édition originale Cidihca 2000). 